\title{
Viscosity and Large-Scale Magnetic Fields from Accretion Disc Dynamos
}

\author{
Christopher A. Tout ${ }^{1}$ \\ Konkoly Observatory of the Hungarian Academy of Sciences, H-1525 \\ Budapest, P.O.B. 67, Hungary.
}

\begin{abstract}
We review those processes associated with accretion discs that are probably influenced by magnetic fields, specifically, accretiondisc viscosity, energy dissipation and jet formation. We consider how magnetic instabilities in the disc can lead to a self-sustaining dynamical dynamo and how this is manifested as magnetohydrodynamic turbulence in numerical simulations. We show that currently these models do not fit with observational constraints imposed by dwarf-nova outbursts. We also show that the drop in ionisation fraction does not lead to the apparently necessary drop in viscosity in quiescent cataclysmic variable discs. Largescale magnetic fields are required to launch and collimate jets form discs. We describe an inverse cascade process that can construct sufficient largescale field from small-scale field generated by a dynamo.
\end{abstract}

\section{Introduction}

Because of the extra complexity that they introduce the effects of magnetic fields have often been ignored in astrophysics. The hope being that their evolution is kinematic in the sense that the bulk flow of material is determined gravitationally and hydrodynamically and the evolution of magnetic fields $\mathbf{B}$ can be determined from the induction equation

$$
\dot{\mathbf{B}}=\nabla \wedge(\mathbf{u} \wedge \mathbf{B})+\eta \nabla^{2} \mathbf{B},
$$

where $\mathbf{u}$ is the determined velocity field and $\eta$ is the, usually very small, magnetic diffusivity. In such cases observed magnetic fields are a very useful diagnostic of the underlying fluid flows.

However it has become increasingly apparent that this is not the case in accretion discs because the Lorentz force, between the field and its associated current density,

$$
\begin{aligned}
\mathbf{F}_{\mathrm{B}} & =\frac{1}{4 \pi}(\nabla \wedge \mathrm{B}) \wedge \mathrm{B} \\
& =\frac{1}{4 \pi}\left[(\mathrm{B} . \nabla) \mathrm{B}-\nabla\left(\frac{1}{2}|\mathrm{~B}|^{2}\right)\right],
\end{aligned}
$$

${ }^{1}$ On leave from the University of Cambridge 
here separated into a magnetic curvature component, that acts when field lines are distorted from a force-free configuration, and an isotropic magnetic pressure, is comparable to the other net forces acting on the disc material.

An accretion disc forms whenever high angular momentum material is accreting on to a central object because material cannot fall on without first losing its angular momentum. If there is any shear viscosity an initial ring of material will spread out into a disc in which the angular momentum is carried outwards by a small fraction of the material while the remainder falls towards the central object. This flow of angular momentum is energetically favourable because the lowest energy state of any rotating object is one of uniform rotation and in a Keplerian disc the outer parts are rotating faster than the inner parts, $\Omega \propto R^{-3 / 2}$, where $\Omega$ is the angular velocity at radius $R$. Accretion discs are indeed found on all scales from the very small, in mass-transferring close binary stars $\left(R_{\mathrm{d}} \approx R_{\odot}\right)$, to the very large, around active galactic nuclei $\left(R_{\mathrm{d}} \approx 1 \mathrm{kpc} \approx 4 \times 10^{10} R_{\odot}\right)$. The source of their viscosity however has long remained a mystery. This is partly because the steady state structure of a disc does not depend on the magnitude of the viscosity and we are limited to observations of time varying discs if we are to have any chance of measuring it.

The fact that discs form at all, for instance in close binary stars where, if left alone, the overflowing material, all with the same specific angular momentum, would just accumulate in a ring, is evidence that molecular viscosity of the disc material is several orders of magnitude too small. Turbulence has always been expected to make a major contribution because turbulent cells can exchange angular momentum through the disc. Shakura \& Sunyaev (1973) parameterised our lack of knowledge of how this may work in terms of an unknown constant $\alpha \leqslant 1$ multiplying the transport by typical turbulent cells of size $H$, the disc pressure scale height, and velocity $c_{s}$, the sound speed,

$$
\nu \approx \alpha H c_{\mathrm{s}} \approx \frac{\alpha c_{\mathrm{s}}^{2}}{\Omega}
$$

where we have used a simple integration of vertical structure to relate $H$ to $\Omega$. However hydrodynamic turbulence alone does not work because it tends to exchange high angular momentum material from the outer parts of the disc with low angular momentum material from the inner parts. This is both contrary to what we want and energetically unfavourable (Tout 1996). Stone \& Balbus (1996) have drawn the same conclusion after considering in more detail angular momentum transport by various turbulent mixing processes.

For cool massive discs (Pacyński 1978) the material itself may become selfgravitating and it is easy to see that the force between two over-dense regions, one further out than the other, tends to accelerate the former while slowing down the latter, transferring angular momentum as required. Attempts to model dynamical instability in protostellar discs (Laughlin \& Bodenheimer 1994) have produced effective viscosities equivalent to $0.01 \leq \alpha \leq 0.03$. However at least discs in binary systems are not self-gravitating.

A third and promising source of viscosity is magnetic fields. Lynden-Bell (1969) proposed that, in discs around active galactic nuclei, they might provide the necessary viscosity. The essence of the effect is that radial field lines in the disc will be bent by differential rotation and the curvature component of 
the Lorentz force, in attempting to straighten the field line, will slow down material at its inner end while accelerating material at its outer end. Again angular momentum is transported outwards. By expanding the curvature term and extracting the mean effect we can write the local magnetic viscosity as

$$
\nu \approx \frac{B_{R} B_{\phi}}{4 \pi \rho \Omega}
$$

or

$$
\alpha \approx \frac{B_{R} B_{\phi}}{4 \pi \rho c_{\mathbf{s}}^{2}},
$$

where $B_{R}$ and $B_{\phi}$ are the radial and azimuthal components of the field and $\rho$ is the density of the material. The magnetic field is here acting dynamically and cannot be treated by the induction equation (1) alone. Although some authors prefer not to use the $\alpha$ prescription when hydrodynamic turbulence is not involved it has become so widely used, particularly in the study of dwarf nova outbursts, that an effective $\alpha$ is still the most useful means of comparison with observations.

We shall discuss the constraints that dwarf nova outbursts place on viscosity and then consider how magnetic instabilities can lead to dynamo action and discuss numerical simulations. We shall also look at how magnetic fields can launch jets from a disc but to do this they must be large-scale and local analyses are insufficient.

\section{Dwarf Novae and the Measurement of Viscosity}

Dwarf novae are cataclysmic variables, very close binaries, with periods up to a few hours. The primary, both the more evolved and the more massive component, is a white dwarf. The secondary is a low-mass, red main-sequence star that is filling its Roche lobe. The orbit is shrinking by loss of angular momentum, probably by magnetic braking of the secondary or gravitational radiation, so that the red dwarf is transferring matter to the white dwarf. This material has sufficient angular momentum that an accretion disc forms. Unlike accretion discs around protostars and active galactic nuclei, this disc is neatly geometrically constrained. It lies in the orbital plane and has a well-defined inner edge at the white dwarf's surface and an outer edge where angular momentum is returned to the orbit via tidal interaction with the secondary. The stream of material flowing from the inner Lagrangian point impacts the disc edge creating a hot spot but the effects do not penetrate far into the disc.

In a typical dwarf nova the disc is observed to brighten by about five magnitudes for a period of days every few months or so. This process can be explained by a disc instability model (Hoshi 1979) in which an annulus of the disc has no stable state for the rate at which material is accreting through the disc. This is the S-curve model. There is a lower stable branch for which, at all surface densities, the flow rate through the annulus is lower than the flow rate into the annulus so that surface density gradually builds up until this lower branch becomes unstable and the annulus flips to a state of the same surface density on an upper branch for which all stable states have flow rates larger than the rate of accretion through the disc. The surface density decreases until it again 
becomes unstable and drops back to the low state. During the outburst the high state propagates through the disc either outwards or inwards. The rise and decay times of the outburst depend on the time taken for the heating and cooling fronts to traverse the disc and can be related to viscosity (Lin, Papaloizou \& Faulkner 1985). They can be fitted with observations if $\alpha \gtrsim 0.1$. The recurrence time for the outburst is related to the rate at which material accumulates at the edge of the disc and the rate at which it flows through the disc in the low state or quiescence. This gives another measure for viscosity (Cannizzo, Shafter $\&$ Wheeler 1988) for which $\alpha \lesssim 0.01$. Thus it appears that $\alpha$ must take two different values $\alpha_{\mathrm{H}}$ and $\alpha_{\mathrm{C}}$ during outburst and quiescence respectively and that

$$
\alpha_{\mathrm{H}} \approx 10 \alpha_{\mathrm{C}} \approx 0.1 \text {. }
$$

\section{Magnetic Instabilities}

In the absence of any other effects the shear in a Keplerian disc will, by induction (equation (1)), shear out any magnetic field into toroidal loops which may have a vertical component, $B_{z}$, but no radial component. The effective viscosity would rapidly decay to zero on the disc dynamical timescale $\tau \approx \Omega^{-1}$. However Balbus \& Hawley (1991) found that vertical field is unstable to perturbations that grow on similar timescales $\left(\tau \approx \Omega^{-1}\right)$ and give rise to a radial field component. This instability arises because any displacement of material distorts the magnetic field creating a torque that can transfer angular momentum outwards in the energetically favourable direction. It can however be stabilized if the field strength is large enough that magnetic tension forces exceed the drag and centrifugal forces on any displaced material.

Balbus \& Hawley (1992) also found that purely azimuthal field is also unstable to a similar magneto-rotational instability. In this case the growth timescale is somewhat longer $\left(\tau \approx 10 \Omega^{-1}\right)$. Such growth rate is similar to that of the most rapidly growing mode of the Parker instability (Horiuchi et al. 1988). This relies on the magnetic buoyancy of embedded magnetic flux. A region threaded by strong magnetic field will have lower density and gas pressure than its surroundings because of the magnetic pressure contributed by the Lorentz force (equation 3). Such regions will rise against gravity. Once a vertical component has appeared material can slip down the field lines towards the central plane. This enhances the density deficit of the rising material which consequently floats up unstably. The gravity vector that is important here is the excess over what is required to balance the centrifugal force in the local Keplerian rotating frame. Although this is nearly perpendicular to the disc it is not precisely aligned with the axis of rotation and so there is a coriolis force on the rising plume which will induce a radial motion and thence a radial field. This alone would lead to viscosity. In addition the induced vertical field will immediately become unstable to the very rapid magneto-rotational pattern.

These various instabilities can lead to generation of all three-dimensional components of magnetic field by tapping the rotational and gravitational energy in the disc. A magnetic dynamo also requires dissipation because without it the topology of the field will remain unchanged and a sufficiently complex pattern will be unwound as much as it is wound up. Even for small diffusivity dissipation can take place by a process of rapid reconnection (Petschek 1964) 
when oppositely directed field components are pushed together. Reconnection at any point leads to field lines which are bent back on themselves. As these rapidly straighten they carry with them associated material creating a density void into which more material carrying oppositely directed field lines is pushed. This is a complex process operating on all scales from the microscopic on which kinetic transport provides the diffusivity to the size of the largest coherent field structures. It is, however, fundamental to dynamo action.

Various combinations of magnetic instabilities and dissipation can be put together to form a self-consistent dynamical dynamo. One such model, devised by Tout \& Pringle (1992), envisages the radial component of field to be generated from the vertical by the Balbus-Hawley instability, the vertical field from the azimuthal by the Parker instability and the azimuthal regenerated from the radial by shear. Reconnection takes place between disordered vertical structures that are formed by these processes and growth is limited by the stabilization of the magneto-rotational action by sufficient vertical field. This model can be criticised on the grounds that, for the Parker instability to become important, the coherence of the field in the disc plane must be at least eight scale heights, while the slower mode of the Balbus-Hawley instability will act on much shorter structures resulting in a generally much less coherent field structure. However it does give rise to a self-regenerating dynamo with $\alpha \approx 0.4$ on average.

An alternative has been considered by Różyczka, Turner and Bodenheimer (1994) in which the radial component is dominantly generated by the coriolis force on the buoyant plumes and in which reconnection is important in all directions. Again a self-regenerating dynamo results but $\alpha \approx 0.01$. Without doubt other combinations would also succeed and so we turn to numerical modelling in order to throw an alternative light on what might be happening physically.

\subsection{Numerical Models}

With current techniques and computational power small regions of a disc can be modelled in three dimensions with considerable success. Two serious problems remain and should be borne in mind. First, the magnetic Reynolds number $\Re_{\mathrm{m}}=L V / \eta$ (where $L$ and $V$ are typical length and velocity scales), which is limited by the smallest modelled scale, or equivalently the grid size relative to the box size, is still too small by ten or more orders of magnitude. Recent increase in the solely hydrodynamic Reynolds number $\Re_{\mathrm{e}}=L V / \nu$ in the modelling of hydrodynamical turbulence indicate that this may do no more than hide small but similar structure from our sight. However the process of reconnection is sufficiently more complicated that this small scale structure may be fundamentally different. It also remains so, that all the magnetic diffusivity in realistic models is artificially provided by grid boundaries or smoothing lengths. Second, the field external to the box has to be enforced by boundary conditions and different boundary conditions do lead to very different results.

Two independent groups of researchers are at the forefront of these investigations. Brandenburg et al. (1995) \& Stone et al. (1996) both produce models in which small and weak magnetic field structures grow to fill the whole of their boxes with self-sustained magnetohydrodynamic turbulence that draws its energy from the shear and which does transport angular momentum in the right direction. These results are encouraging because they show that a dynamical 
dynamo can indeed operate. However they are disappointing in that it is rather difficult to produce $\alpha \gtrsim 0.01$. Although this is compatible with our observed $\alpha_{\mathrm{C}}$ it is not with the, perhaps better constrained, $\alpha_{\mathrm{H}}$ required for dwarf-nova outbursts. Brandenburg et al. (1996)) do point out that increasing the numerical resolution does tend to increase $\alpha$ but probably not by enough. It is also possible to generate large values of $\alpha$ by imposing a fixed vertical field component in the boundary conditions at the top and bottom of the disc. However in practice, as we shall discuss later, it is very difficult to maintain any external vertical field threading a rotating disc so that these simulations are probably unrealistic. We should also note that it is very difficult to determine exactly what boundary condition should be used because of the exponential decay in density and the uncertain behaviour at the disc surface where we believe outflows and jets originate.

\subsection{Energy Dissipation}

Since reconnection and dissipation need not take place at the same location in the disc as that at which the viscosity acts a magnetic mechanism could be used to explain the anomalous spectra of some apparently steady-state cataclysmicvariable accretion discs. Far enough from the inner edge, viscous theory, in which the energy generation is local, predicts that the effective temperature of the disc surface $T_{\text {eff }} \propto R^{-3 / 4}$ and from this that the spectral energy distribution with wavelength $F_{\lambda} \propto \lambda^{-7 / 3}$ (Lynden-Bell 1969). The temperature profiles (from eclipse mapping - see Horne in these proceedings) and spectra of dwarf novae in outburst fit these theoretical predictions well but those of nova-like variables, which are supposed to be in a constant state of outburst, have flatter temperature profiles and redder spectra (Rutten, van Paradijs \& Tinbergen 1992). IUE observations of the anti-dwarf nova TT Ari, that swaps irregularly between high and low states, seem to indicate that its spectrum is redder some time after entering a high state than it is initially. This led Tout, Pringle \& la Dous (1995) to postulate that the anomalous energy dissipation from a steady state dynamo leads to the redder spectrum and that the dynamo takes a relatively long time to build up to this steady state. However for most nova-like variables there exists a much more satisfactory explanation in terms of the concavity of the disc and the viewing angle (Smak 1994) that does not require any anomalous energy dissipation. Unfortunately the artificial dissipation in numerical models excludes them from illuminating this issue.

\subsection{Low Ionisation and Dwarf-Nova Outbursts}

If magnetic fields are responsible for viscosity then one commonly held hope was that low ionisation $0.1<\rho_{\mathrm{i}} / \rho<0.9$, where $\rho_{\mathrm{i}}$ is the partial density of ionised species, might be the cause of the comparatively low $\alpha_{\mathrm{C}}$. Regös (1996a) has investigated this possibility by examining the effect of reduced ionisation on each of the processes described at the beginning of this section. She finds that neither the Parker nor Balbus-Hawley instabilities are grossly affected and that the major change is to the reconnection rate, because the conductivity $\sigma=1 / \eta \propto \rho_{\mathrm{i}} / \rho$, but that even here the effect is small because the reconnection timescale

$$
\tau_{\text {rec }} \propto \log _{e} \Re_{\mathbf{m}} \propto \log _{e} \sigma .
$$


Reanalysing the Tout and Pringle model in the light of this she finds that $\alpha$ actually increases slightly in response to enhanced dynamo activity. In the case of Różyczka et al.'s model $\alpha$ would decrease slightly but certainly not by a factor of ten. Regös' result is supported by the numerical work of Brandenburg et al. (1995) who do not find a significant effect either. For the much lower ionisation fractions $\left(\rho_{\mathrm{i}} / \rho \approx 10^{-10}\right)$ encountered in protostellar discs the effect is more involved and Regös (1996b) has calculated that the effect on the instabilities cancels that of the increased reconnection rate and so $\alpha$ does not differ from the fully ionised case.

In the light of this it is appropriate to consider an alternative mechanism for dwarf-nova outbursts (Armitage, Livio \& Pringle 1996). This relies on the Balbus-Hawley instability stabilizing when vertical fields are strong. A dynamo operating during a high state provides a viscosity which allows material to flow out of the disc faster than it is replenished. As a result the disc shrinks until its thickness is less than the critical wavelength for the instability to grow, $\lambda_{\text {crit }} \propto B_{z} / \Omega$. At this point the dynamo switches off or is severely inhibited. Material is now replenished faster than it flows out of the disc and builds up until either the thickness exceeds $\lambda_{\text {crit }}$ or the field has decayed sufficiently for the dynamo to restart and initiate a new high state. The observational details of this model have yet to be analysed.

\section{Jets}

Another phenomenon which magnetic fields in accretion discs can explain rather well is the launching of outflows and jets and the collimation of the latter. Jets or outflows appear to be associated with all objects in which there is believed to be an accretion disc (Livio - these proceedings). If the launching process is universal then the central object or boundary layer cannot be important because black holes have neither an accessible surface nor a boundary layer. Nor can thermal acceleration play a significant role because protostellar jets cool too quickly.

On the other hand a large scale magnetic field threading the disc and inclined away from the rotation axis by about $30^{\circ}$ can effectively centrifugally accelerate material that that threads on to the field lines at the disc surface. This angle is rather critical. If it is less the potential increases outwards along the field line so that nothing flows along it while if it is more the potential gradient close to the surface is so steep that all the disc material will rapidly escape. This launching angle may be self regulating but this has not been established.

Pringle (1993) has shown that the strength of the large scale field need not be very large. The back pressure in a jet with flow rate $\dot{M}_{\text {jet }}$ and speed $v_{\text {jet }}=R_{\text {jet }} \Omega$ from within a radius $R_{\text {jet }}$ can be equated with the magnetic pressure in the large-scale field $B_{J}$.

$$
P_{\mathrm{jet}} \approx \frac{\dot{M}_{\mathrm{jet}} v_{\mathrm{jet}}}{R_{\mathrm{jet}}^{2}} \approx \frac{\dot{M}_{\mathrm{jet}} \Omega}{R_{\mathrm{jet}}} \equiv \frac{B_{\mathrm{j}}^{2}}{4 \pi} .
$$

And the gas pressure in the disc, that can be related, through $\alpha$, to the accretion rate $\dot{M}_{\text {acc }} \approx \rho R H v_{r}$, where $v_{r} \approx \nu / R$ is the radial drift velocity, can be equated 
with the magnetic pressure of a dynamo generated field $B_{\mathrm{D}}$.

$$
P_{\mathrm{gas}} \approx \rho c_{\mathrm{s}}^{2} \approx \frac{\rho \nu \Omega}{\alpha} \approx \frac{\dot{M}_{\mathrm{acc}} \Omega}{H \alpha} \equiv \frac{B_{\mathrm{D}}^{2}}{4 \pi \alpha} .
$$

From which

$$
\frac{B_{\mathrm{J}}^{2}}{B_{\mathrm{D}}^{2}} \approx \frac{\dot{M}_{\mathrm{jet}}}{\dot{M}_{\mathrm{acc}}} \frac{H}{R_{\mathrm{jet}}} \ll 1
$$

because the disc is thin and the rate of mass outflow is intuitively expected and observed to be somewhat smaller than the accretion rate.

Although the large-scale field need only be small it is still not obvious how it can arise. Lubow, Papaloizou \& Pringle (1994a) have shown that it is difficult to drag in the field of an ambient medium or secondary star by advection with the accretion flow if similar processes are responsible for viscosity and magnetic diffusivity. As field is advected in, the lines will be bent and they will tend to slip out owing to the resulting tension. Lubow et al. showed that to achieve the launching angle

$$
\frac{R}{H} \frac{\eta}{\nu} \leq 0.88
$$

This is far from satisfied when $\eta \approx \nu$, as they are if produced by similar mixing processes. This will certainly be the case if viscosity is the result of magnetohydrodynamic turbulence. However an alternative scenario for disc accretion (Königl 1989), in which angular momentum is carried away from all radii by a small fraction of the mass accelerated along field lines anchored in the disc, avoids this problem because it has no need for viscosity at all. It does however rely on creating a suitable launching angle at every radius (see Lubow, Papaloizou \& Pringle 1994b and Königl 1996 for further discussion of this point).

It is even more difficult to thread the disc with the field of the central object, a field that will not even exist in the case of a black-hole accretor. Either the field will be so strong that it disrupts the disc altogether (Ghosh \& Lamb 1979) or it will be weak enough to be dragged around differentially relative to the star (at least everywhere except at a single radius) and will be wound up azimuthally. The ensuing strong curvature force will expel the field radially from the disc (Bardou \& Heyvaerts 1996).

\subsection{Inverse Cascade}

Tout \& Pringle (1996) have investigated a third possibility, that the small-scale (coherence length $\approx H$ ) but relatively strong dynamo generated field can somehow generate the large scale but significantly weaker component. The mechanism that they propose is that short loops of flux emerging from the surface of the disc are pushed around by both random motions, from the ensuing turbulence, and the shear in the disc and reconnect at their footpoints when oppositely directed feet get sufficiently close to oneanother. Recall that such reconnection, as well as being inevitable, is a necessary component of the dynamo. In this way two loops of short radial extent can combine to form a single one of longer radial extent. Equally a long loop and a short one can combine to form a single shorter one. 
With a constant rate for footpoint reconnection Tout and Pringle have shown analytically in one dimension that the distribution of loop lengths

$$
n(L) \propto \frac{1}{L^{2}},
$$

where $n(L) \delta L$ is the number of loops with length between $L$ and $L+\delta L$. They found the same distribution after a long time in a two-dimensional simulation that included shear.

In order to accelerate an outflow sufficiently, the loops must have a radial extent similar to the local radius of the disc and integrating over all loops longer than this gives an estimate of the large-scale field strength

$$
B_{\mathrm{J}} \propto \int_{R}^{\infty} n(l) d l \propto \frac{1}{R} .
$$

Similarly the strength of the small-scale, dynamo-generated field,

$$
B_{\mathrm{D}} \propto \int_{H}^{\infty} n(l) d l \propto \frac{1}{H}
$$

so that

$$
\frac{B_{\mathrm{J}}}{B_{\mathrm{D}}} \approx \frac{H}{R}
$$

which can be combined with equation (10) to show that

$$
\frac{\dot{M}_{\mathrm{jet}}}{\dot{M}_{\mathrm{acc}}} \approx \frac{H}{R} \approx 0.01 \rightarrow 0.3
$$

depending on the system. This result is consistent with observations and provokes more detailed analysis. Unfortunately full global numerical simulations are still out of reach (see Stone - these proceedings).

\section{Conclusions}

We have shown that magnetic fields can be dynamically important in accretion discs: they can provide the necessary viscosity; they may be responsible for anomalous energy dissipation; and they can launch jets and outflows. They may even collimate the jets (Spruit 1996). All magnetic field configurations in a differentially rotating disc appear to be unstable and various dynamo mechanisms that tap the differential rotation, and as such are viscous, can be envisaged. Numerical simulations confirm that dynamo action does operate but always degenerates to sustained magnetohydrodynamic turbulence. They do not result in a large enough viscosity nor can they say anything about energy dissipation nor much about large scale field structures, if any exist. An inverse cascade mechanism can generate the large-scale but relatively weak magnetic field that is needed to centrifugally accelerate jets and outflows from the disc.

Acknowledgments. The author thanks Mario Livio, the IAU and the NSF for contributing towards his expenses. 


\section{References}

Armitage, P. J., Livio, M. \& Pringle, J. E. P. 1996, ApJ, 457, 332

Balbus, S. A. \& Hawley, J. F. 1991, ApJ, 376, 214

Balbus, S. A. \& Hawley, J. F. 1992, ApJ, 392, 662

Bardou, A. \& Heyvaerts, J. 1996, A\&A, 307, 1009

Brandenburg, A., Nordlund $\AA$., Stein, R. F. \& Torkelsson, U. 1995, ApJ, 446, 741

Brandenburg, A., Nordlund §., Stein, R. F. \& Torkelsson, U. 1996, ApJ, 458, L45

Cannizzo, J. K., Shafter, A. W. \& Wheeler, J. C. 1988, ApJ, 333, 227

Ghosh, P. \& Lamb, F. K. 1979, ApJ, 232, 259

Horiuchi, T., Matsumoto, R., Hanawa, T. \& Shibata, K. 1988, PASJ, 40, 147

Hoshi, R. 1979, Prog. Theor. Phys., 61, 1307

Königl, A. 1989, ApJ, 342, 208

Königl, A. \& Wardle, M. 1996, MNRAS, 279, L61

Laughlin G. \& Bodenheimer, P. 1994, ApJ, 436, 335

Lin, D. N. C., Papaloizou, J. C. B. \& Faulkner, J. 1985, MNRAS, 212, 105

Lubow, S. H., Papaloizou, J. C. B. \& Pringle, J. E. 1994a, MNRAS, 267, 235

Lubow, S. H., Papaloizou, J. C. B. \& Pringle, J. E. 1994b, MNRAS, 268, 1010

Lynden-Bell, D. 1969, Nature, 223, 690

Pacyński, B. 1978, Acta Astr., 28, 91

Petschek, A. G. 1964, in The Physics of Solar Flares, W. N. Hess, Greenbelt: NASA, 425

Pringle, J. E. 1993, in Astrophysical Jets, D. Burgarella, M. Livio \& C. P. O'Dea, Cambridge University Press, 1

Regős, E. 1996a, MNRAS, in press

Regős, E. 1996b, MNRAS, in press

Różycka, M., Turner, N. J. \& Bodenheimer, P. 1994, MNRAS276, 1179

Rutten, R. J. M., van Paradijs, J. \& Tinbergen, J. 1992, A\&A, 260, 213

Shakura, N. I. \& Sunyaev, R. A. 1973, A\&A, 24, 337

Smak, J. 1994, Acta Astr., 44, 265

Spruit, H. C. 1996 in Evolutionary Processes in Binary Stars, R. A. M. J. Wijers, M. B. Davies \& C. A. Tout, Dordrecht: Kluwer, 249

Stone, J. M. \& Balbus, S. A. 1996, ApJ, 464, 364

Stone, J. M., Hawley, J. F., Gammie, C. F. \& Balbus, S. A. 1996, ApJ, 463, 656

Tout, C. A. 1996, in Cataclysmic Variables and Related Objects, A. Evans \& J. H. Wood, Dordrecht: Kluwer, 97

Tout, C. A. \& Pringle, J. E. 1992, MNRAS, 259, 604

Tout, C. A. \& Pringle, J. E. 1996, MNRAS, 281, 219

Tout, C. A., Pringle, J. E. \& la Dous C. 1993, MNRAS, 265, L5 


\section{Discussion}

S. Balbus: I wouldn't give up just yet on the magnetic instability producing a value of $\alpha$ significantly above 0.01 . While it is true that some simulations do indeed saturate at this level, others give higher values. (For instance starting off with a vertical field gives a value several times higher). The size of the computational box makes a difference. In some cases, there are even solutions that run away! Until these various numerical effects have been sorted out, it is best not to grasp at some particular value of $\alpha$ as an upper limit.

C. Tout: I have not given up and would rather hope these comments have been encouraging for future investigations. [It is both worrying and interesting that initial conditions are important].

S. Chakrabarti: Unlike stars (like the Sun) where both the radiative core and convective envelope exist, it is not easy to form magnetic loops in discs by anchoring much of the field underneath the convective zone. But in discs [Chakrabarti \& De Silva (1994)] such loops cannot be formed unless the disc is close to isothermal. Thus, I have a problem with pictures of discs which are embedded by loops of fields.

C. Tout: It is true that gas pressure supported discs are relatively stable to the Parker instability that would produce deep rooted loops of flux. Indeed closed buoyant loops of flux may simply float off into space carrying mass and energy with them. However, there is evidence for hot coronae above disc surfaces similar to that in the sun which does seem to be heated by anchored flux loops. I think the question of loop generation remains open.

A. Konigl: Magnetic flux transport through the disk could proceed efficiently if the effective viscosity were not based on the same physical process as the magnetic diffusivity. One attractive possibility is that the angular momentum removal is effected by the transported open magnetic field lines themselves. A self consistent calculation along these lines in presented in the poster by Contopoulos and Konigl.

C. Tout: Indeed if such a flow can be set up and maintained in a stable manner these problems would be overcome. 